\title{
Mitigation of Edge Localised Modes with magnetic perturbations in ASDEX Upgrade
}

\author{
W. Suttrop J. C. Fuchs, R. Fischer, L. Giannone, A. Herrmann, \\ R. M. McDermott, M. Maraschek, A. Mlynek, H. W. Müller, \\ P. Lang, T. Pütterich, M. Rott, T. Vierle, E. Viezzer, E. Wolfrum, and \\ the ASDEX Upgrade Team \\ Max-Planck-Institut für Plasmaphysik, EURATOM Association, D-85740 Garching, \\ Germany
}

\begin{abstract}
The first stage of a significant enhancement of the ASDEX Upgrade experiment with invessel coils for non-axisymmetric magnetic perturbations is now operational. First experiments have shown that ELM mitigation can be achieved using various perturbation field configurations with toroidal mode numbers $n=1,2,4$. The main access criteria is the plasma edge pedestal density to exceed a threshold, which takes the lowest value of about $60 \%$ of the Greenwald density for resonant $|n|=1$ perturbations. In H-mode plasmas, mode locking or error field-induced magnetic islands are generally not observed. Due to the low local shear of the plasma magnetic field in the vicinity of the perturbation coils around the outboard midplane, the magnetic perturbation is resonant simultaneously on several rational surfaces. It is hypothesised that the existence of image currents on these surfaces ensures good shielding of the error field in the confined plasma.
\end{abstract}

Key words: Tokamaks, ASDEX Upgrade, MHD control, Edge Localised Modes PACS: $52.55 \mathrm{Fa}, 52.55 . \mathrm{Rk}$, 52.55.Tn

\section{Introduction}

The ASDEX Upgrade (AUG) experiment is currently being enhanced with a set of in-vessel saddle coils and supporting tools for MHD control [1]. The first stage of this project, a set of 16 off-midplane coils capable of producing a small nonaxisymmetric perturbation field (radial field $B_{r} \sim 10^{-3} B_{t}$ ), has been implemented as described in Refs. [2] and [3]. For the 2011 experimental campaign, a first subset of eight coils (four above and four below the midplane) have been available. The full set of eight coils above and eight coils below the midplane has become 
operational for the 2012 campaign. So far, experiments have been conducted with DC currents provided by pre-existing grid-commutated thyristor bridge converters (two independent four quadrant circuits). A complementary modular and economical AC-capable power supply is in preparation [4].

In view of ITER needs, physics experiments so far have mainly targeted ELM mitigation [5,6], effects on High-confinement mode (H-mode) transport barrier [7,8], $3 \mathrm{D}$ effects on equilibrium and divertor magnetic configuration $[9,10], \mathrm{H}$-mode access threshold [11], scrape-off layer properties [12], and fast particle transport [13]. In this paper, we concentrate on the effects of perturbation field configuration on ELM mitigation.

\section{ELM mitigation phenomenology}

A regime for ELM mitigation by $n=2$ magnetic perturbations has been found soon after commissioning the first eight saddle coils [5]. With application of DC currents, it is possible to change type-I ELMs into a benign form of ELMs with high repetition rate and energy loss typically reduced by a factor of 10 or more. The inner divertor remains completely detached at all times and the inner target heat flux essentially vanishes. The outer divertor peak heat flux is reduced by typically a factor of 4 or larger, and splitting of strike lines due to the non-axisymmetry of the perturbation can be seen by infrared thermography of the targets [10].

The transition to small ELMs is induced by raising the edge density above a threshold, which from data obtained so far can be described as a pedestal density of $65 \%$ of the Greenwald density $n_{\mathrm{GW}}$ [14]. At the threshold, small ELMs occur in between, and gradually replace, large ELMs. In this regime, there is no gradual evolution of ELM losses, as e.g. observed in JET [15]. ELM mitigation is compatible with cryogenic pellet injection [16] to fuel the plasma core up to $1.5 \times n_{\mathrm{GW}}$, while -unlike in plasmas without magnetic perturbation-no large ELMs are triggered by the pellets.

Subsequently, we discuss the effects of magnetic perturbation field configuration on ELM mitigation, in extension of a previous conference paper [17].

\section{Magnetic perturbation field structure}

The non-axisymmetric, doubly periodic magnetic perturbation field can be described in terms of toroidal $(n)$ and poloidal $(m)$ mode numbers, i.e. the magnetic spectrum. The saddle coils produce a radial field of order $B_{r} \sim 10^{-3} B_{t}$ at the plasma surface close to the coils positions, which drops off rather quickly with increasing distance to the coils. The eight coils of each toroidal row are positioned at regular 


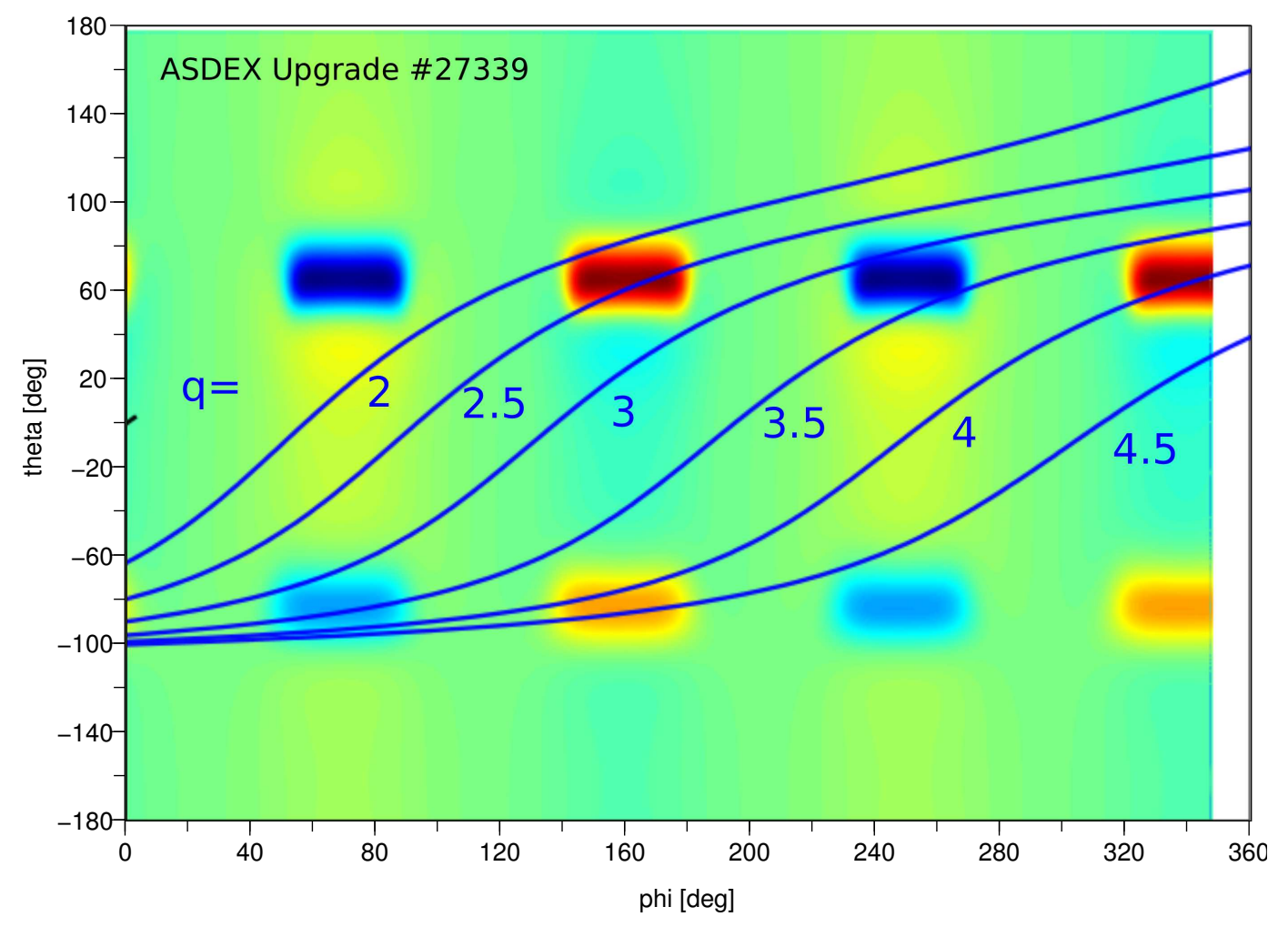

Fig. 1. Normal field on the unfolded nominal $q=-2$ surface (colour contours), overplotted with field lines at different rational surfaces.

toroidal angle intervals, so the toroidal mode number spectrum is dominated by the fundamental mode of the coil current pattern, $n= \pm 1, \pm 2$ or \pm 4 with harmonics $l \times n$ ( $l$ odd ordinal number) and aliasing components ( $4 \pm n$ etc $)$.

Magnetic surfaces are resonant with the perturbation field, if the safety factor of that surface $q=m / n$, or in other words, if field lines close into themselves after $m$ toroidal and $n$ poloidal passes around the torus. By convention, for the standard AUG field and plasma current directions used here $\left(B_{t}<0, I_{p}>0\right), q<0$, so a resonance occurs for $m / n<0$. The mode number $(m, n)$ spectrum is point-symmetric, and here we choose to describe it in the $m>0$ half-space, so that a resonance can occur for $n<0$.

The poloidal truncation of the coils results in a broad $m$-spectrum, however the existence of two toroidal rows allows to vary the resonant field component. This is important, since the nature of expected physics effects on the plasma differs for resonant and non-resonant perturbation fields.

The magnetic plasma configuration has a profound effect on the occurrence of resonances. As an example, we consider the diverted plasma discharge AUG \#27339 (lower single null) with toroidal field $B_{t}=-2.5 \mathrm{~T}$, plasma current $I_{p}=1.2 \mathrm{MA}$, and edge safety factor $q_{95}=-3.4$. Eight saddle coils are operated with a coil current $I=960$ A (with five windings of each coil, corresponding to $4.8 \mathrm{kA} \times$ turns) 


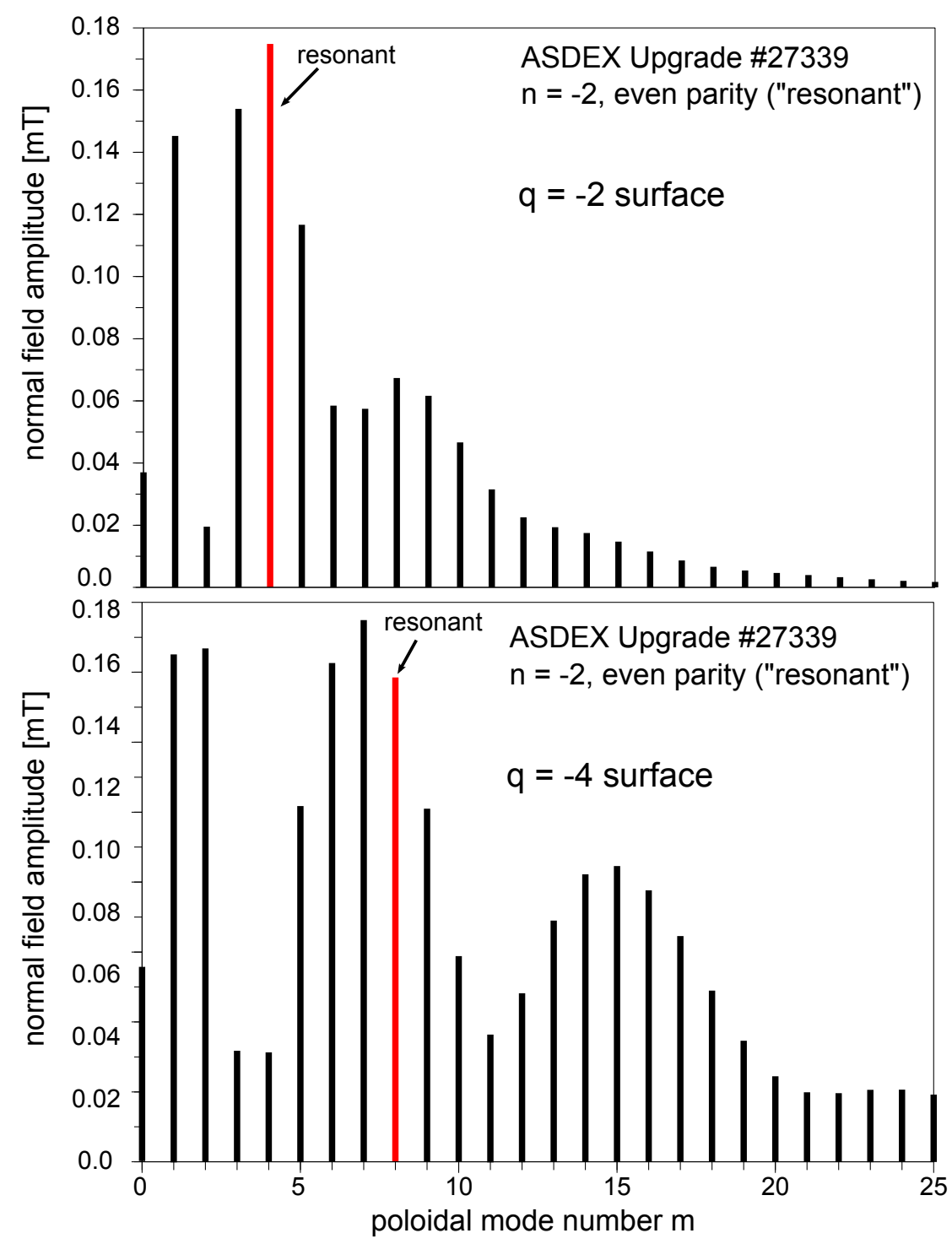

Fig. 2. Poloidal mode number spectrum $B_{n}(m)$ [T] for $n=-2$ magnetic perturbation, calculated for the $q=-2$ (top) and $q=-4$ (bottom) surfaces.

to produce an $n= \pm 2$ error field with "even" up/down parity, i.e. same direction of the radial field in upper and lower coil rings. Fig. 1 shows, as colour contours, the normal component of the vacuum perturbation field, calculated on the $q=-2$ flux surface, as reconstructed from the unperturbed (i.e. axisymmetric) magnetohydrodynamic force equilibrium, plotted vs. toroidal $(\phi)$ and poloidal $(\theta)$ geometrical angles of the axisymmetric torus. Outboard midplane is at $\theta=0$, plasma top at $\theta=90^{\circ}$ and bottom at $\theta=-90^{\circ}$. Blue and red colours denote opposite sign (direction) of the normal field. Overplotted are field lines (at arbitrary toroidal position) at different half-integer safety factor, $q=-2 \ldots-4.5$. Due to vanishing axisymmetric poloidal field at the magnetic limiter (active $X$-line at $\theta \approx-100^{\circ}$ ) the magnetic field is mainly in toroidal direction in the vicinity of the lower and upper X-lines. A surface with rational $q$ is spanned by mutually unconnected field lines. As can be seen in Fig. 1, there are field lines that intersect regions of either only positive (say, 


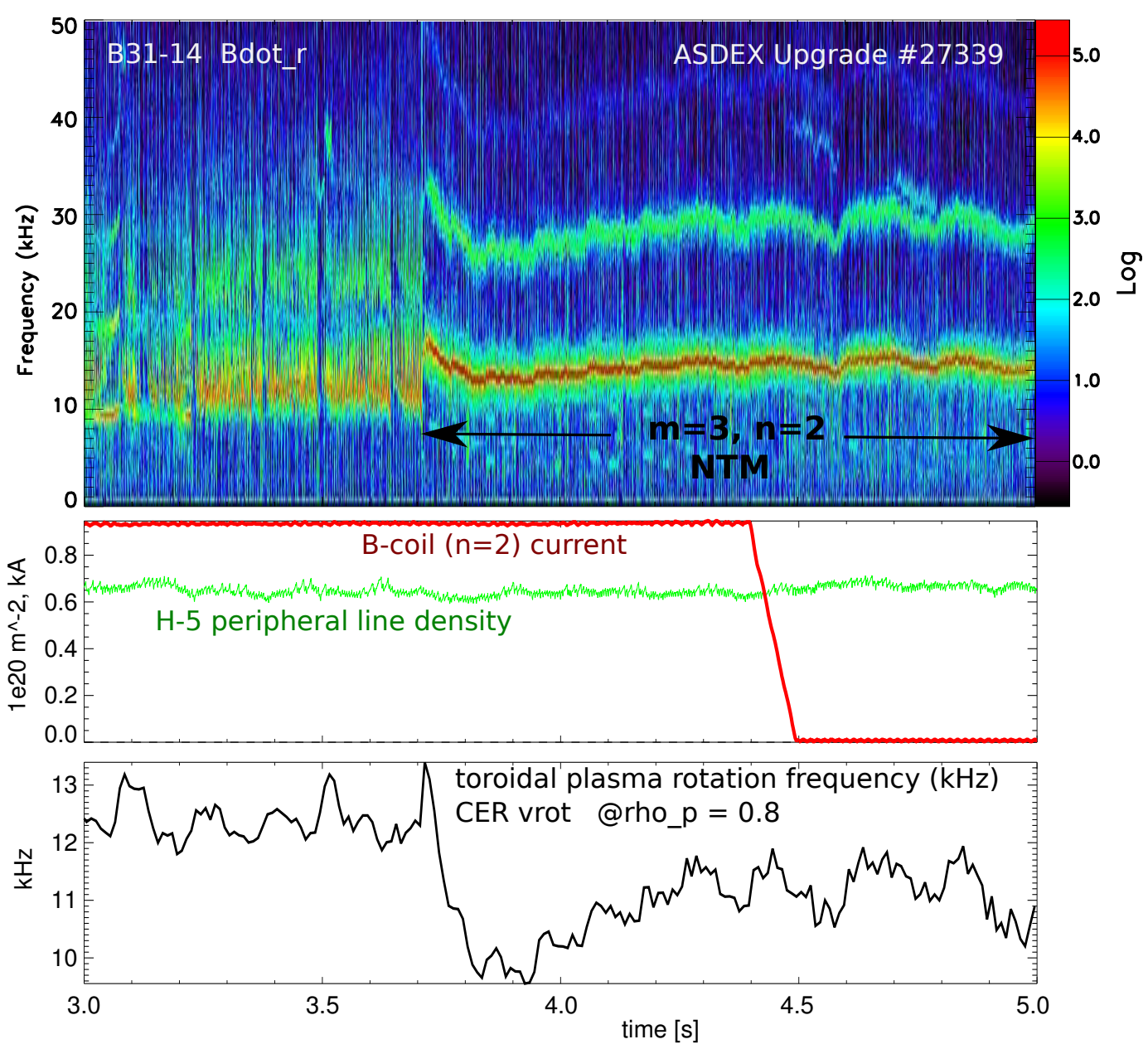

Fig. 3. Time traces of AUG pulse \#27339.

blue) or only negative (red) normal perturbation field. Resonant effects can occur, for example induction of a field-aligned helical current in the presence of plasma flows. Conversely, if the current direction in one of the two coil rows is reversed (“odd" parity), resonant effects are minimised.

At the outboard midplane, in between the two rows of saddle coils, the local magnetic shear is small, and the resonance condition is either met or not met simultaneously for suitable rational surfaces. In other words, the global shear originates to a large extent from the regions at the plasma bottom and top, i.e. diversion and elongation produced by the plasma shaping currents. It is interesting to note that the H-mode edge bootstrap current density, however important locally for edge stability, does not significantly influence the resonance condition on internal flux surfaces because the total edge bootstrap current is small (order of 10\%) compared to the total plasma and shaping currents.

In a modal representation, the effects of a non-circular plasma (and broad spectrum of the external perturbation) can be described as coupling between poloidal spectral components [18]. However, the main effects can more easily be visualised by 
considering a "local" mode spectrum, separately for each rational flux surface in the plasma (half-integer $q$ for $n=-2$ in our example). In a straight field line angle system $\left(\phi^{*}, \theta^{*}\right)$, conveniently the one with $\phi^{*}=\phi$, the poloidal spectrum can be obtained by a Fourier transform in $\theta^{*}$ direction for any $n$ component of choice. Since the transformation $\theta^{*}(\theta)$ depends on the metrics of the flux surface, this "local" spectrum is different for each resonant surface. Fig. 2 shows the poloidal spectrum for our example at the $q=-2$ and $q=-4$ surfaces. Resonant components $(m=q n)$ are highlighted in red colour; they are at or near a maximum on both surfaces (and all resonant surfaces in between), and their amplitude is of the order of $10^{-4} B_{t}$, one order below the peak field in front of the coils. Reversal of the up/down parity from "even" to "odd" shifts the spectral minima into the resonance and hence allows to produce virtually entirely non-resonant perturbations everywhere. The non-resonant field components are similarly strong in both cases.

Many resonant surfaces exist, in particular in the high shear region near the separatrix due to the poloidal field null at the $\mathrm{X}$-line. This can be expected to become important for the screening of the perturbation field by induced helical image currents in the plasma [19]. The calculation of these screening currents is not straightforward and involves considering forced reconnection and magnetic islands that interact non-linearly with the surrounding rotating plasma [20]. However, it can be expected that calculations for circular limiter plasmas (with only one resonant surface) systematically underestimate the field shielding effects.

An experimental hint is given by the example pulse, \#27339. Strong auxiliary heating, Neutral Beam Injection $P_{\mathrm{NBI}}=12.5 \mathrm{MW}$, Ion Cyclotron Radio Frequency heating $P_{\mathrm{ICRF}}=3 \mathrm{MW}$, and Electron Cyclotron Resonance Heating $P_{\mathrm{ECRH}}=1.6 \mathrm{MW}$, does not only allow access to high-confinement mode but also gives rise to a plasma beta-driven neo-classical tearing mode (NTM). Fig. 3 shows time traces of saddle coil current, plasma density and toroidal plasma rotation frequency, measured by charge exchange recombination spectroscopy (CER) on a heating beam at a normalised flux radius of $\rho_{p}=0.8$ as well as a spectrogram of a magnetic probe showing the NTM island rotation. The $m=3, n=2$ tearing mode appears at $t=3.7 \mathrm{~s}$, accompanied by slowing down of the plasma rotation, while the saddle coils are operated at constant current. The saddle coil current is ramped down from $t=4.4-4.5 \mathrm{~s}$, but the removal of the magnetic perturbation apparently does not influence the MHD mode activity nor the plasma fluid rotation speed. Assuming vacuum perturbation field and a helical current of $I=10 \mathrm{kA}$ associated with the NTM at the $q=3 / 2$ surface, the resonant $I \times B_{n}$ torque exceeds the neutral beam torque input by two orders of magnitude. Yet no discernible change of island or plasma rotation due to the change of saddle coil current is seen. This is typical of AUG H-mode pulses where effects of the error field on plasma rotation are mostly very weak [6]. 

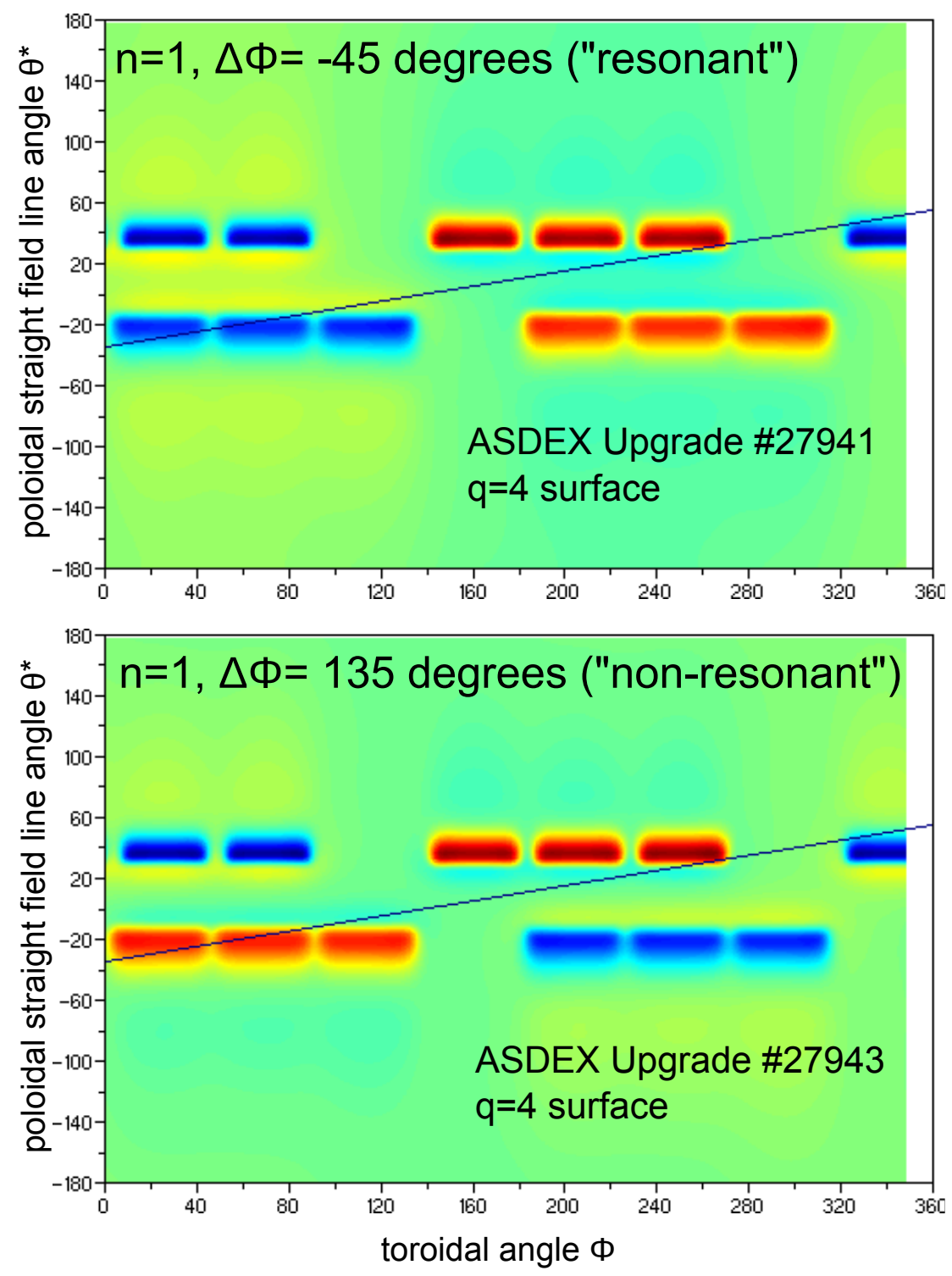

Fig. 4. Normal field on $q=-4$ surface for a coil current perturbation aimed to approximate an $n=1$ perturbation.

\section{ELM mitigation with $|n|=1$ magnetic perturbations}

Magnetic perturbations with $|n|=1$ can be approximated even with only one power supply circuit. Fig. 4 shows the normal field on the unfolded $q=-4$ surface in straight field line coordinates, again with a field line for comparison. The toroidal phase angle $\Delta \phi$ of upper vs. lower coil ring is adjusted (in $45^{\circ}$ increments) to maximise the resonant component for pulse \#27941 $\left(\Delta \phi=-45^{\circ}\right)$ and to minimise it for pulse \#27943 $\left(\Delta \phi=135^{\circ}\right)$. The resulting $m$ spectra are shown in Fig. 5 . Thus, the resonant field component $(m=4, n=-1)$ on the $q=-4$ surface, is varied by more than a factor of 2.5 . 

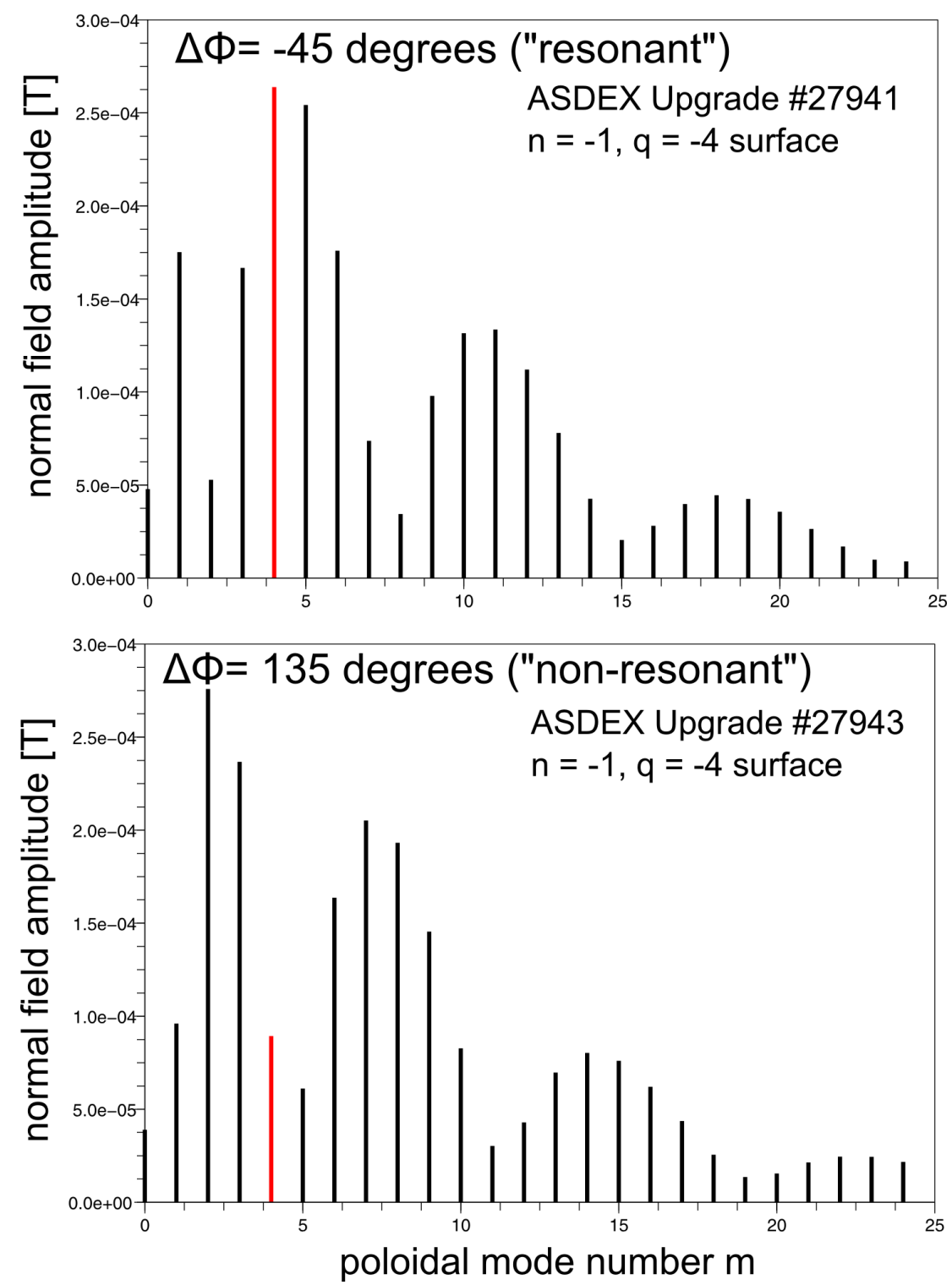

Fig. 5. Poloidal mode number $(m)$ spectra of the $n=-1$ toroidal mode of the perturbation shown in Fig. 4.

Fig. 6 shows time traces of these discharges and a control experiment (\#27942) with saddle coils off. Apart from the saddle coil configuration, the plasma parameters are identical: Fuelling by feed forward gas puff, $B_{t}=-2.45 \mathrm{~T}, I_{p}=0.8 \mathrm{MA}$, low triangularity shape, and edge safety factor $q_{95}=-5.1$. These plasmas can be directly compared with a similar configuration reported in Ref. [6] for $n=2$ perturbations. During a phase with DC saddle coils current $(I=900 \mathrm{~A})$ applied, the gas rate is ramped up linearly to probe the ELM mitigation threshold. The appearance of type-I ELMs is unambiguously detected as large spikes in the outer divertor currents, measured with a shunt resistor of one outer divertor plate sections. For both optimum resonant and non-resonant perturbations type-I ELMs disappear completely at a certain time during the gas scan. The plasma density (shown in Fig. 

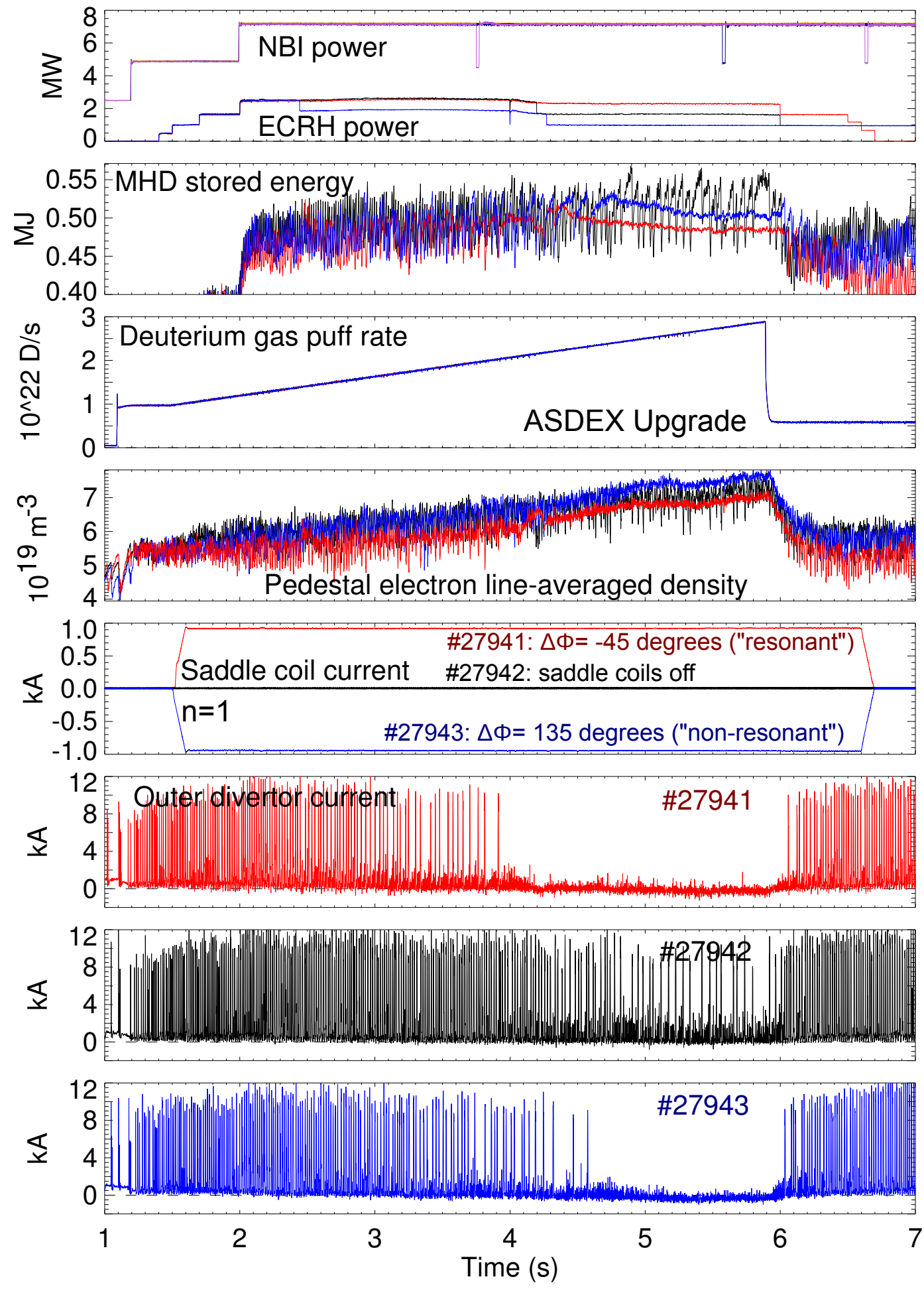

Fig. 6. Time traces of AUG pulses \#27941 ( $|n|=1$ resonant perturbation), \#27943 (non-resonant) and \#27942 (control pulse with saddle coils off).

6 is the line-averaged density from a peripheral DCN interferometer chord) differs: With non-resonant field, it is about $10 \%$ above that with resonant field and that of the reference discharge. The onset of ELM mitigation occurs at an edge density of 
$6.2 \times 10^{19} \mathrm{~m}^{-3} \sim 60 \% n_{\mathrm{GW}}$ for the resonant case and $7.1 \times 10^{19} \mathrm{~m}^{-3} \sim 69 \% n_{\mathrm{GW}}$ for the non-resonant case. In the control case with saddle coils off, type-I ELMs persist even at higher pedestal density, $7.3 \times 10^{19} \mathrm{~m}^{-3} \sim 71 \% n_{\mathrm{GW}}$, ultimately at reduced repetition frequency.

\section{$5 \quad$ ELM mitigation with $|n|=4$ magnetic perturbations}

Eight coils in toroidal direction allow to produce perturbations with $|n|=4$ fundamental and essentially no toroidal subharmonics. Since resonant components have high poloidal mode number and therefore decay on short radial scales, it is interesting to see if ELMs can be mitigated.

Figure 7 shows the most promising discharge with $|n|=4$ made in AUG so far. The gas rate is adjusted to obtain a pedestal density close to the density threshold found previously for $|n|=2$ perturbations $\left(65 \% n_{\mathrm{GW}}\right)$. Other plasma parameters are similar to the $|n|=1$ experiment discussed in the previous section. Upper and lower saddle coil rows are powered separately by a four quadrant converter each. This allows, in a single pulse, to operate one coil row alone (upper or lower row), and to flip the upper/lower phase between even and odd parity, which are non-resonant and resonant, respectively, for this discharge. Type-I ELMs are completely suppressed only for non-resonant perturbation and, to some extent, with current only in the lower coil row. Properties of the ELM mitigation phases are very similar to the observations with $|n|=2$ fields: The inner divertor power (measured by infrared thermography on the target) essentially vanishes due to (temporally and spatially) complete detachment, and the outer divertor power (area integrated heat flux) drops to close to the previous inter-ELM level.

The pedestal density responds very little to the changes of the perturbation field structure and remains close to the empirical $|n|=2$ threshold value. It should be noted that in discharges (not shown here) with lower gas puff $\left(\Gamma=1 \times 10^{22} \mathrm{D} / \mathrm{s}\right)$, ELM mitigation was not observed and with higher gas puff $\left(\Gamma=3 \times 10^{22} \mathrm{D} / \mathrm{s}\right)$, only small ELMs occurred regardless of whether saddle coils were operated or not. In all cases there was little variation of the plasma density, which shows that the $|n|=4$ ELM mitigation window is rather restricted.

\section{Summary and Conclusion}

In summary, ELM mitigation is observed in ASDEX Upgrade by application of magnetic perturbations with the newly installed set of $2 \times 8$ in-vessel saddle coils, using a variety of configurations with $|n|=1,2,4$ and a varying phase angle of upper and lower toroidal ring to probe the importance of resonant components. 

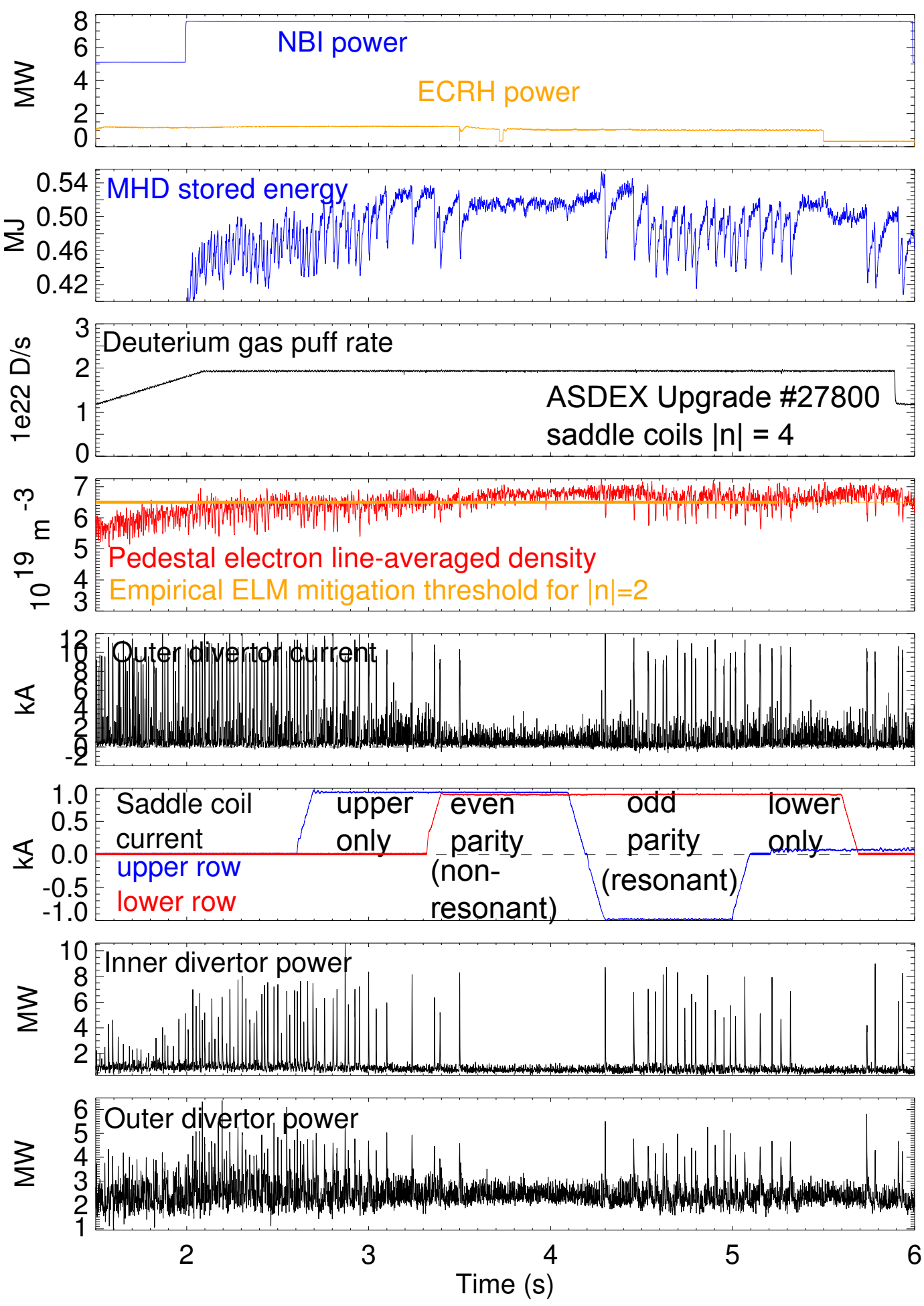

Fig. 7. Time traces of AUG pulse $\# 27800(|n|=4)$ with time intervals of resonant, non-resonant and single coil row perturbations (upper or lower row).

The ELM mitigation effect does not seem to rely on the resonant perturbation field, however the plasma density reacts somewhat to the field configuration. In particular at $|n|=1$, the edge density higher by about $10 \%$ for entirely non-resonant pertur- 
bation compared to the cases with resonant and no perturbation field. This finding contrasts somewhat with observations of density "pump-out" during resonant perturbations in other experiments $[15,21,22]$. A clear density reduction is observed in ASDEX Upgrade H-modes with resonant perturbations at lower plasma density, albeit not in combination with type-I ELM suppression.

Application of $|n|=1$ resonant magnetic perturbations seem to reduce the threshold density for ELM mitigation by about $10 \%$ compared to $|n|=2$ and no operational penalty such as vulnerability to locked modes seems to be encountered for H-mode plasmas. ELM mitigation has been found in plasmas with different heating methods, different momentum input and hence, different plasma rotation velocities. If penetration of the magnetic perturbation into the core plasma, beyond the edge pedestal, was essential for ELM mitigation, one would expect a dependence on plasma rotation. Geometrical considerations suggest that image currents on many resonant surfaces can be an essential ingredient for field screening, however a quantitative study of error field attenuation (and field penetration) for these AUG pulses is still missing. Experimentally, there is no indication for significant magnetic islands produced by the external field. Even pre-existing tearing modes typically do not lock to the error field in H-mode plasmas.

As a main restriction, ELM mitigation is found only at relatively high pedestal density, $n / n_{\mathrm{GW}} \geq 60 \%$. In AUG, this corresponds to relatively large pedestal collisionalities, $v_{i}^{*} \geq 1.0$, although this does not seem to be a collisionality boundary [6]. Attempts to reproduce the low collisionality DIII-D ELM suppression scenario [21] in AUG are being made, so far without success.

\section{References}

[1] W. Suttrop, O. Gruber, S. Günter, D. Hahn, A. Herrmann, et al., Fusion Engineering and Design 84 (2009) 290.

[2] T. Vierle, B. Streibl, M. Rott, U. Seidel, A. Herrmann, et al., Fusion Engineering and Design 84 (2009) 1928.

[3] M. Rott, U. Seidel, B. Streibl, W. Suttrop, T. Vierle, et al., Fusion Engineering and Design 84 (2009) 1653.

[4] M. Teschke, W. Suttrop, M. Rott, and the ASDEX Upgrade Team, Power inverter design for ASDEX Upgrade saddle coils, this conference.

[5] W. Suttrop, T. Eich, J. C. Fuchs, S. Günter, A. Janzer, et al., Phys. Rev. Lett. 106 (2011) 225004.

[6] W. Suttrop, L. Barrera, A. Herrmann, R. M. McDermott, T. Eich, et al., Plasma Physics and Controlled Fusion 53 (2011) 124014. 
[7] R. Fischer, C. Fuchs, B. Kurzan, R. McDermott, T. Pütterich, et al., in Europhysics Conference Abstracts (CD-ROM, Proc. of the 38th EPS Conference on Plasma Physics, Strasbourg, France, 2011), edited by A. Becoulet, T. Hoang, and U. Stroth, volume 35G of ECA, page P1.072, Geneva, 2011, European Physical Society.

[8] E. Viezzer, T. Pütterich, G. D. Conway, R. Dux, T. Happel, et al., High-accuracy characterization of the edge radial electric field at ASDEX Upgrade, submitted to Nucl. Fus.

[9] J. Fuchs, T. Eich, R. Fischer, L. Giannone, A. Herrmann, et al., in Europhysics Conference Abstracts (CD-ROM, Proc. of the 38th EPS Conference on Plasma Physics, Strasbourg, France, 2011), edited by A. Becoulet, T. Hoang, and U. Stroth, volume 35G of ECA, page P1.090, Geneva, 2011, European Physical Society.

[10] T. Lunt, Y. Feng, M. Bernert, A. Herrmann, P. de Marné, et al., Nuclear Fusion 52 (2012) 054013.

[11] F. Ryter, R. Fischer, B. Kurzan, T. Pütterich, S. K. Rathgeber, et al., L-H transition in the presence of magnetic perturbations in ASDEX Upgrade, 13th H-mode workshop, Oxford 2011, submitted to Nucl. Fus.

[12] H. W. Müller, T. Lunt, W. Suttrop, T. Eich, R. Fischer, et al., Modification of Scrapeoff Layer Transport and Turbulence by Non-axisymmetric Magnetic Perturbations in ASDEX Upgrade, submitted to J. Nucl. Mat.

[13] M. García-Muñoz, S. Äkäslompolo, O. Asunta, J. Boom, X. Chen, et al., Fast-ion redistribution and loss due to edge perturbations in the ASDEX Upgrade, DIII-D and KSTAR tokamaks, IAEA Fusion Energy Conference 2012, submitted to Nucl. Fus.

[14] M. Greenwald, J. L. Terry, S. M. Wolfe, S. Ejima, M. G. Bell, et al., Nucl. Fusion 28 (1988) 2199.

[15] Y. Liang, H. R. Koslowski, P. R. Thomas, E. Nardon, B. Alper, et al., Phys. Rev. Lett. 98 (2007) 265004.

[16] P. Lang, W. Suttrop, E. Belonohy, M. Bernert, R. McDermott, et al., Nuclear Fusion 52 (2012) 023017.

[17] W. Suttrop, L. Barrera Orte, R. Fischer, C. Fuchs, R. McDermott, et al., in Europhysics Conference Abstracts (CD-ROM, Proc. of the 39th EPS Conference on Plasma Physics, Stockholm, Sweden, 2012), page P2.092, Geneva, 2012, European Physical Society.

[18] R. Fitzpatrick, Phys. Plasmas 17 (2010) 112502.

[19] M. Bécoulet, F. Orain, P. Maget, N. Mellet, X. Garbet, et al., Nuclear Fusion 52 (2012) 054003.

[20] R. Fitzpatrick, Plasma Phys. Controlled Fusion 54 (2012) 094002.

[21] T. E. Evans, M. E. Fenstermacher, R. A. Moyer, T. H. Osborne, and J. G. Watkins, Nuclear fusion 48 (2008) 024002.

[22] A. Kirk, E. Nardon, R. Akers, M. Bcoulet, G. D. Temmerman, et al., Nuclear Fusion 50 (2010) 034008. 